\title{
SU(N) Grand Unification with Several Quark-Lepton Generations*
}

P. H. Frampton

Department of Physics The Ohio State University

Columbus, Ohio 43210
This report was prepared as an account of work sponsored by the United States Government. Neither the United States nor the United States Department of Energy, nor any of their employees, nor any of their contractors, subcontractors, or their employees, makes any warranty, express or implied, or assumes any legal liability or responsibility for the accuracy, completeness or usefulness of any information, apparatus, product or process disclosed, or represents that its use would not infringe privately owned rights.

\begin{abstract}
Procedures are given for embedding SU(5) into SU(N) such

that several SU(5) generations remain as ordinary-mass fermions.

Examples with an arbitrary number of generations occur already

for SU(7). All models have only quarks and leptons, without

quixes or quaights.

${ }^{*}$ Supported in part by the U.S. Department of Energy.
\end{abstract}




\section{DISCLAIMER}

This report was prepared as an account of work sponsored by an agency of the United States Government. Neither the United States Government nor any agency Thereof, nor any of their employees, makes any warranty, express or implied, or assumes any legal liability or responsibility for the accuracy, completeness, or usefulness of any information, apparatus, product, or process disclosed, or represents that its use would not infringe privately owned rights. Reference herein to any specific commercial product, process, or service by trade name, trademark, manufacturer, or otherwise does not necessarily constitute or imply its endorsement, recommendation, or favoring by the United States Government or any agency thereof. The views and opinions of authors expressed herein do not necessarily state or reflect those of the United States Government or any agency thereof. 


\section{DISCLAIMER}

Portions of this document may be illegible in electronic image products. Images are produced from the best available original document. 
The simplest possible grand unification ${ }^{1}$ of QCD with the Weinberg-Salam model is based on the group SU(5). Applications of this model to determine the weak mixing angle, ${ }^{2}$ and certain fermion mass ratios, ${ }^{3}$ are encouraging. One major shortcoming of the theory is that each generation of quarks and leptons is put into its own irreducible representation in a trivial fashion; different generations are not related by the gauge group. Here we consider embedding $\mathrm{SU}(5) \subset \mathrm{G}=\mathrm{SU}(\mathrm{N}), \mathrm{N}>5$, such that a non-trivial flavor group emerges. We choose $\mathrm{SU}(\mathrm{N})$ only because Nature appears to have a predilection for special unitary groups. Our results appear more economical than those of Ref. 4 .

We make the usual general assumptions., First, we allow only singlets and triplets under SU(3) of color, thus avoiding quixes (6) and quaights (8). This requires ${ }^{5}$ that we take only the totally antisymmetric fundamental representations which we designate $[\mathrm{N}, \mathrm{m}], 1 \leq \mathrm{m} s(\mathrm{~N}-1)$, for $\mathrm{m}$ tensor indices (or boxes in the Young Tableaux). Each generation of left-handed fermions in SU(5) is thus

$$
[5,2]+[5,4] \equiv \underset{\sim}{10}+5_{\sim}^{*}
$$

and $[\mathrm{N}, \mathrm{m}]$ decomposes into $\mathrm{SU}(5)$ according to

$$
\begin{aligned}
{[\mathrm{N}, \mathrm{m}] \rightarrow } & \left(\begin{array}{c}
\mathrm{N}-5 \\
\mathrm{~m}
\end{array}\right) \underset{\sim}{1}+\left(\begin{array}{c}
\mathrm{N}-5 \\
\mathrm{~m}-1
\end{array}\right) \underset{\sim}{5}+\left(\begin{array}{l}
\mathrm{N}-5 \\
\mathrm{~m}-2
\end{array}\right) \underset{\sim}{10} \\
& \left(\begin{array}{c}
\mathrm{N}-5 \\
\mathrm{~m}-3
\end{array}\right) \underset{\sim}{10}+\left(\begin{array}{l}
\mathrm{N}-5 \\
\mathrm{~m}-4
\end{array}\right) \underset{\sim}{5^{*}}+\left(\begin{array}{c}
\mathrm{N}-5 \\
\mathrm{~m}-5
\end{array}\right) \underset{\sim}{1} .
\end{aligned}
$$

Second, we assume the particular set $\{\mathrm{m}\}$ we take for the left-handed fermions certains equal number of $3_{c}$ and ${\underset{\sim}{c}}_{c}^{*}$ for the quarks. This ensures non-chiral color. Therefore, if the SU(5) content is 


$$
[\mathrm{N},\{\mathrm{m}\}] \rightarrow v(1) \underset{\sim}{1}+v(5) ._{\sim}^{5}+v(10) 10+v\left(10^{*}\right) 10^{*}+v\left(5^{*}\right) 5_{\sim}^{*}
$$

we require that

$$
v(5)+v(10)=v\left(5^{*}\right)+v\left(10^{*}\right) \text {. }
$$

Third, we impose renormalizability in the sense that there are no triangle anomalies. In the sU(5) language, this requirement coincides.with Eq. (4): reality under SU(3) color coincldes with absence of anomalies.' In SU(N) language, one requires

$$
\sum_{\{m\}} A[N, m]=0
$$

with the anomaly number given by ${ }^{6}$

$$
A[N, m]=-A[N, N-m]=\frac{(N-2 m)(N-3) !}{(N-m-1) !(m-1) !}
$$

Fourth, we assume ${ }^{4}$ that the number of ordinary-mass fermion su(5) families is given by

$$
g=v(10)-v\left(10^{*}\right)
$$

which also equals $\left(\nu\left(5^{*}\right)-\nu(5)\right)$ by Eq. (4). For each representation one therefore has a generation increment from Eq.(2) of

$$
\Delta \mathrm{g}[\mathrm{N}, \mathrm{m}]=-\Delta \mathrm{g}[\mathrm{N}, \mathrm{N}-\mathrm{m}]=\left(\begin{array}{l}
\mathrm{N}-5 \\
\mathrm{~m}-2
\end{array}\right)-\left(\begin{array}{l}
\mathrm{N}-5 \\
\mathrm{~m}-3
\end{array}\right) \text {. }
$$

We note that if $g \neq 0$, the representation for the left-handed fermions is automatically complex under the flavor $S U(2)_{L} \otimes U(1)$ subgroup of $S U(5)$. Fifth, we require ${ }^{7}$ that within the set $\{\mathrm{m}\}$ the coefficients $\mathrm{C}_{\mathrm{m}}$ in $\{m\}=\sum_{m} C_{m}[m]$ do not all have a common factor. This excludes trivial replication of a single generation or of subsets of generations. 
In order to construct models, our procedure is now to tabulate the $A[N, m]$ and $\Delta g[\hat{N, m}]$ for $1 \leq m \leq(N-1)$ with a given $N$. We then look for linearly independent combinations $\{\mathrm{m}\}^{\prime}$ satisfying

$$
\begin{aligned}
& \sum_{\{m\}^{\prime}} A[N, m]=0 \\
& \sum_{\{m\}} \Delta g[N, m] \neq 0 .
\end{aligned}
$$

The number of such linearly-independent combinations is given by (N-3) for odd $N,(N-4)$ for even $N$ since for even $N, A[N, N / 2]=\Delta g[N, N / 2]=0$. ThIs number falls into $\left[\frac{1}{2}(N-3)\right]([x]=$ integer part of $x)$ pairs of complex conjugates having $\Delta g>0$ and $\Delta g<0$, respectively. These combinations, each of particular $\Delta \mathrm{g}$, are then readily assembled to make models of definite total generation number, $g$.

The procedure is best illustrated by the simplest examples $N=6$ and 7 . For SU(6):

$\begin{array}{rlllll}\mathrm{m} & =1 & 2 & 3 & 4 & 5 \\ \mathrm{~A} & =+1 & +2 & 0 & -2 & -1 \\ \Delta \mathrm{g} & =0 & 1 & 0 & -1 & 0 .\end{array}$

we have the anomaly-free combination $\left(D=\right.$ dimension; $D[N, m]=\left(\begin{array}{l}N \\ m\end{array}\right)$ )

$$
\{\mathrm{m}\}=([2]+2[5]) ; \quad \Delta g=1 ; \quad D=27
$$

\begin{tabular}{|c|c|c|c|c|}
\hline$m=1$ & 2 & 3 & 4 & 5 \\
\hline$A=+1$ & +3 & +2 & -2 & -3 \\
\hline$\Delta \mathrm{g}=0$ & 1 & 1 & -1 & -1 \\
\hline
\end{tabular}

and its complex conjugate. The only model is uninteresting since it has $g=1$. For $\mathrm{SU}(7)$ : 
There are now two independent combinations

$$
\begin{aligned}
& \{m\}_{1}=([2]+3[6]) ; \quad \Delta g=1 ; \quad D=42 \\
& \{m\}_{2}=([3]+2[6]) ; \quad \Delta g=1 ; \quad D=49
\end{aligned}
$$

and this complex conjugates. Here we can make models for all $g$, including the six interesting values $8 \geq g \geq 3$, respecting asymptotic freedom and accomodatIng the known fermons. For $g=3$, say, there are two models with dimension 133 and 140. The lower dimension is explicitly

$$
\{m\}=(2[7,2]+[7,3]+8[7,6]) ; \quad g=3 ; \quad D=133
$$

The second model corresponds to the alternative partitioning of $3=2+1=1+2$. Models for $g=4,5$ : . are similarly possible. Thus, already in SU(7) we have models with arbitrary generation number. The same procedure can be followed for $S U(N), N>7$, quite easily.

At this point, we should indicate the slight difference between this and Ref. 4 which finds in SU(11) the candidate model

$$
\{m\}=([11,4]+[11,8]+[11,9]+[11,10]) ; \quad g=3 ; \quad D=561 .
$$

and, no. others for $3 \leq \mathrm{g} \leq 8$ based on the assumption that any given irreductble representation $[\mathrm{m}]$ can occur in the set $\{\mathrm{m}\}$ not more than once. We have relaxed this assumption, regarding it as more advantageous to minimize $D$ than the number of irreducible representations.

There now seems to appear much arbitrariness but for given $g$, and the minimal value of $\mathrm{N}=7$, we achieve uniqueness by minimizing the number $\mathrm{D}$ of left-handed fermions. Thus one finds the sequence $g=3, D=133 ; g=4, D=175 ; g=5, D=217$; $g=6, D=259 ; g=7, D=301 ; g=8, D=343$. In each case (15g) fermtons are the 
residual light SU(5) generations and the remainder are expected to have superheavy mass comparable to the grand unification scale.

of course, the mystery of the gauge heirarchy remains. Also the uneasy feeling remains concerning the fact that ordinary matter is comprised only of the minority fraction debris, while most quarks and leptons have completely inaccessible masses. Nevertheless, a study of the symmetry breaking in a model like my Eq.(14) should lead to new testable relations between generations; the difficulty here is first that the $\mathrm{SU}(7)$ group appears to $\mathrm{mix}$ the light fermions with the superheavy ones of unknown experimental properties, but perhaps the latter can be somehow factored out? Second, one must invent a plausible simplicity criterion for the breaking pattern itself given the plethora of possible irreducible representations? Our purpose here was only to note a simplifying possibility for the matter fields. 


\section{$\underline{\text { References }}$}

1. .H. Georg1 and S. L. Glashow, Phys. Rev. Lett. $\underline{32}, 438$ (1978).

2. H. Georg1, H. Quinn, and S. Weinberg, Phys. Rev. Lett. 33, 451 (1974).

3. A. Buras, J. Ellis, M. K. Galllard, and D. V. Nanopoulos, Nucl. Phys. B135, 66 (1978); P. H. Frampton, S. Nandi, and J. J. G. Scanio, Ohio State University preprint c00-1545-253 (1979).

4. H. Georg1, Harvard preprint HUTP-79/A013 (1979).

5. M. Gell-Mann, P. Ramond, and R. Slansky, Revs. Mod. Phys. 50, 721 (1978).

6. J. Banks and H. Georg1, Phys. Rev. D 14, 1159 (1976); S. Okubo, Phys. Rev. D. 16,3528 (1977).

7. This is where we differ from Ref. 4; see later in the text. 